Ecology, 0(0), 2019, e02774

(C) 2019 by the Ecological Society of America

\section{Killifish eggs can disperse via gut passage through waterfowl}

Giliandro G. Silva, ${ }^{1,6}$ Vinícius Weber, ${ }^{1}$ Andy J. Green, ${ }^{2}$ Pedro Hoffmann, ${ }^{1}$ Vanessa S. Silva, ${ }^{3}$ Matheus V. Volcan, ${ }^{4}$ Luis Esteban K. Lanés, ${ }^{4}$ Cristina Stenert, ${ }^{1}$ Martin Reichard ${ }^{5}$, And LeONARdo Maltchik ${ }^{1}$

Manuscript received 25 March 2019; revised 22 April 2019; accepted 3 May 2019. Corresponding Editor: John Pastor.

${ }^{1}$ Laboratory of Ecology and Conservation of Aquatic Ecosystems, Universidade do Vale do Rio dos SinosUNISINOS, 950 Unisinos Avenue, São Leopoldo, Rio Grande do Sul, Brazil.

${ }^{2}$ Department of Wetland Ecology, Estación Biológica de Doñana (EBD-CSIC), C/Américo Vespucio 26, Sevilla 41092 Spain.

${ }^{3}$ Parque Zoológico da Fundação Zoobotânica do Rio Grande do Sul, BR-116 Road, km 252, Sapucaia do Sul, Rio Grande do Sul, Brazil.

${ }^{4}$ Laboratory of Ichthyology, Instituto Pró-Pampa-IPPampa, 1242 Uruguay Street, Pelotas, Rio Grande do Sul, Brazil.

${ }^{5}$ Czech Academy of Sciences, Institute of Vertebrate Biology, Květná 8, Brno, 60365 Czech Republic.

${ }^{6}$ E-mail: giliandrog@gmail.com

Citation: Silva, G. G., V. Weber, A. J. Green, P. Hoffmann, V. S. Silva, M. Volcan, L. E. K. Lanés, C. Stenert, M. Reichard, and L. Maltchik. 2019. Killifish eggs can disperse via gut passage through waterfowl. Ecology 00(00):e02774. 10.1002/ecy.2774

Key words: Anatidae; annual fish; diapause; endozoochory; killifishes; waterbirds; wetland.

How freshwater fish arrived in remote lakes and pools has been much debated. It has often been suggested that fish eggs are dispersed when they are attached to the feet, feathers, or bills of waterbirds (a form of ectozoochory). However, a recent thorough review failed to provide solid empirical evidence for such dispersal (Hirsch et al. 2018). We discovered an alternative mode to waterbird-facilitated dispersal of fish eggs - inside the alimentary system of coscoroba swan (form of endozoochory) - and experimentally demonstrated that killifish eggs from bird feces were capable of continuing their development after spending over $30 \mathrm{~h}$ inside a swan (Fig. 1).

Killifish are cyprinodontiform fishes that live in marginal habitats, from isolated desert pools to shallow mangrove swamps, including temporary ponds. Some species of killifish independently evolved dry-resistant eggs that are adapted to survive in desiccated pool substrate (Furness et al. 2015). Dry-resistant eggs are crucial for persistence of fish populations in isolated habitats that desiccate annually. Unusually large flooding events are considered the main dispersal strategy of annual killifish across landscapes, though presence of remote populations in high-altitude areas and population genetic patterns provide indirect evidence for unusual dispersal events (Bartáková et al. 2013), and suggest the existence of alternative dispersal modes.

While investigating endozoochory of plants and invertebrates in southern Brazil (Parna Lagoa do Peixe and Santa Vitória do Palmar), we discovered one egg of an annual killifish (Austrolebias sp.) and six fish egg envelopes (chorions) in 2 of 27 coscoroba swan (Coscoroba coscoroba) fecal samples collected in the field (Fig. 1A; Appendix S1: Table S1). The feces were collected fresh from grass and were not in contact with soil or water. The egg was apparently intact and contained a welldeveloped embryo (Fig. 1B; Appendix S1: Fig. S1), but our samples were frozen prior to inspection, so egg viability could not be tested. Hence, we experimentally tested whether killifish eggs can survive passage through waterfowl (Anatidae) guts and if endozoochory can be a mode of fish egg dispersal.

We used 650 killifish eggs from captive breeding (Appendix S1) of two annual killifish species (350 Austrolebias minuano, Fig. 1C, and 300 Cynopoecilus fulgens, Fig. 1D) that are not closely related but coexist in the Coastal Plain of Rio Grande do Sul, Brazil (31 ${ }^{\circ} 17^{\prime} \mathrm{S}$; $\left.51^{\circ} 5^{\prime} \mathrm{W}\right)$, where droppings with a fish egg and chorions were previously found. The experimental eggs were in diapause I (600 eggs) or diapause II (50 eggs of $A$. minuano) developmental stages (Podrabsky et al. 2017) that are the most frequent for eggs collected in the field (Reichard and Polačik 2019). We mixed the eggs into a standard 120 -g ration of corn-based food provided to three captive coscoroba swans in the Parque Zoológico da Fundação Zoobotânica do Rio Grande do Sul (PZFZB) and allowed them to feed on it during a period of $4 \mathrm{~h}$ (7:00 11:00 a.m.). The ration was then removed, but birds could still feed on aquatic macrophytes present in their enclosure. The following days, the swans were submitted to a normal feeding routine, identical to the experimental day, except that no eggs were mixed into their morning ration. At six time intervals $(0,6,20,24,30$, and $48 \mathrm{~h})$ after removal of the mixed ration, we collected a total of 55 droppings (mean \pm standard deviation weight: $33.13 \pm$ $14.03 \mathrm{~g}$ ) and analyzed their content for the presence of eggs (Appendix S1).

We recovered five viable killifish eggs in four droppings (Appendix S1: Table S2). Three eggs were of 


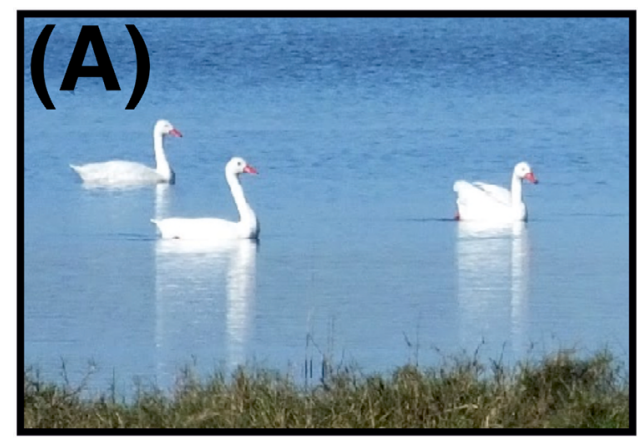

\section{(B)}

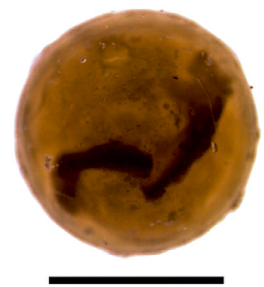

\section{(C)}
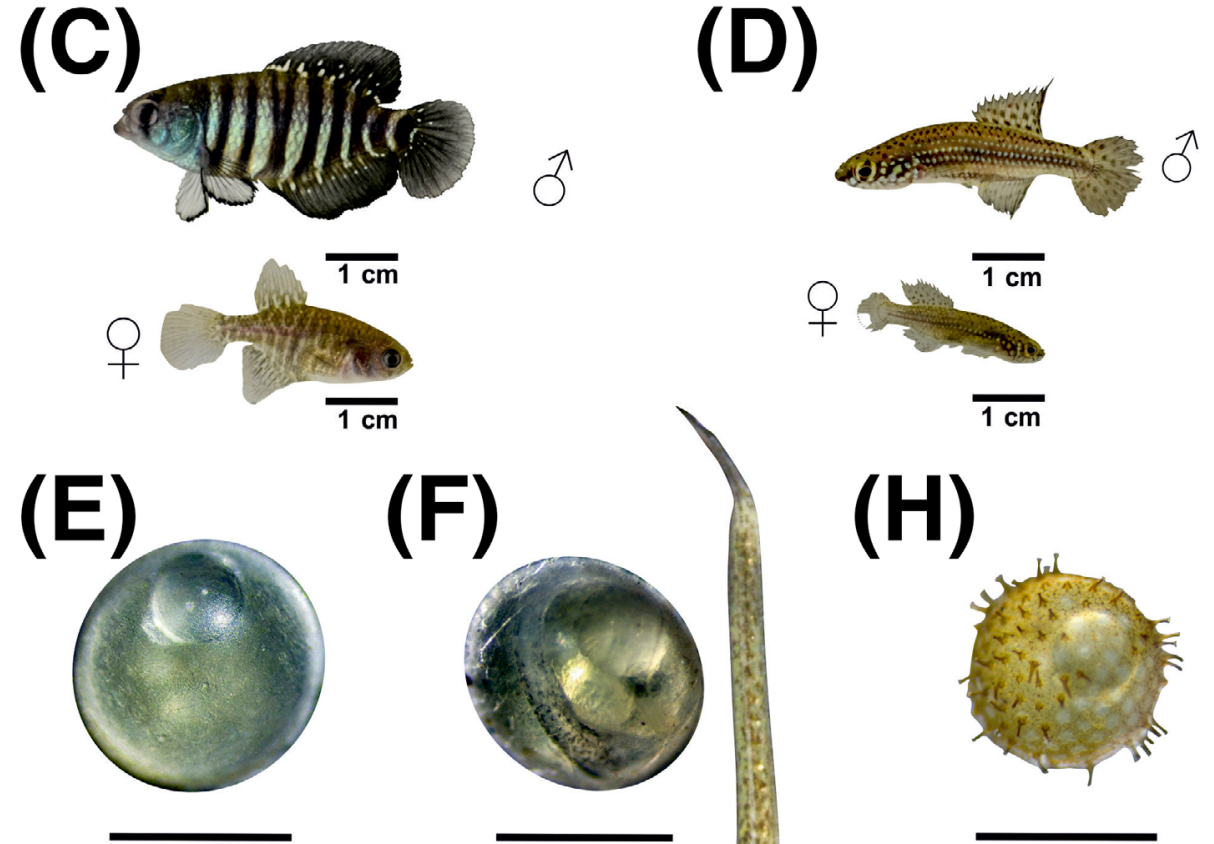

(F)

(H)

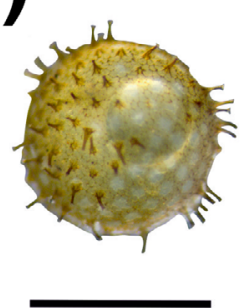

\section{(G)}
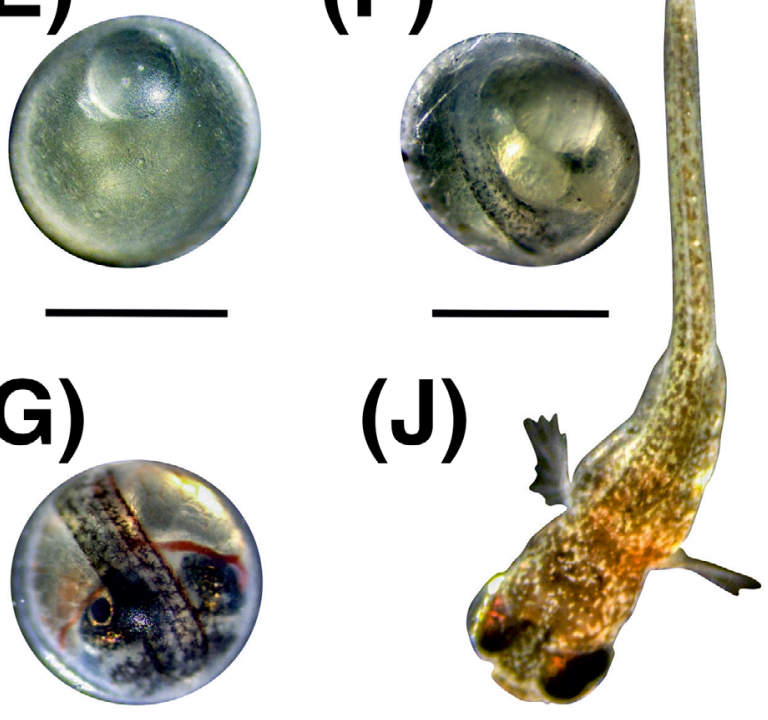

(I)

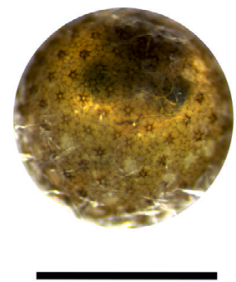

FIG. 1. Study animals and eggs that survived waterfowl gut passage. (A) Coscoroba swans in the Lagoa do Peixe; (B) egg of Austrolebias sp. recovered from swan feces collected in the field; (C) male and female Austrolebias minuano; and (D) male and female Cynopoecilus fulgens. Egg Aust 2 (A. minuano) when recovered from an experimental dropping at (E) diapause I after its development to $(\mathrm{F})$ diapause II and $(\mathrm{G})$ diapause III. Egg Cyno 1 (C. fulgens) when recovered from an experimental dropping in (H) diapause I and (I) between diapause I and II. (J) Freshly hatched juvenile A. minuano, after developing from egg Aust 3 that survived passage through the coscoroba swan digestive tract. Scale bars refer to $1 \mathrm{~mm}$ for eggs and hatched fish and $1 \mathrm{~cm}$ for adult fish.

A. minuano (Fig. 1E-G) and two of $C$. fulgens (Fig. $1 \mathrm{H}$, I). Two A. minuano eggs (in one dropping) were collected immediately after ration withdrawal (time: $0 \mathrm{~h}$ ) and must have gone through the swan digestive system within $4 \mathrm{~h}$. A single egg of $C$. fulgens was collected after $20 \mathrm{~h}$. Two eggs, one of each species, were collected $48 \mathrm{~h}$ 
after ration withdrawal and must have been in the digestive system for at least $30 \mathrm{~h}$. We also collected 15 chorions in nine droppings (Appendix S1: Table S2). The experimental procedures were authorized by the UNISINOS Ethic Committee (PPECEUA03.2018) and Brazilian Agency ICMBio (Sisbio 63602-1), and supervised by a veterinarian and biologists from PZFZB.

After their removal from droppings, we observed embryonic development in three of the five eggs recovered, and one of these hatched. One egg (Aust 3, A. minuano) was recovered in diapause II stage and developed to diapause III in 35 d (Appendix S1: Fig. S2), then successfully hatched $49 \mathrm{~d}$ after removal from the dropping (Fig. 1J; Video S1). The embryo hatched in 54 min after immersion in water. Two eggs (Aust 2 and Cyno 1) were recovered in diapause I stage and developed beyond diapause II (Appendix S1: Table S2), but later died from fungal infections. The egg Aust 2 died at ready-to-hatch postdiapause III stage following $63 \mathrm{~d}$ of development (Appendix S1: Fig. S3). The egg Cyno1 died at postdiapause II stage following $49 \mathrm{~d}$ of development (Appendix S1: Fig. S4). The other two eggs (Aust 1 and Cyno 2) died from fungal infection within the first week of incubation (Appendix S1: Table S3). Mortality of eggs from fungal infection is common in the laboratory and unlikely to be due to ingestion by waterfowl. We recovered 48 eggs of $A$. minuano and 65 of $C$. fulgens from the remains of the mixed ration that had not been ingested by waterfowl $(5.629 \mathrm{~g})$. The proportion of live eggs egested by birds corresponded to $1 \%$ of the ingested eggs.

Dispersal of other aquatic taxa that typically coexist with fish in small lakes is well described, with abundant evidence of avian endozoochory of specialized dispersal stages such as seeds, resting eggs of zooplankton, and statoblasts of bryozoans (Green et al. 2016, Lovas-Kiss et al. 2018). However, propagules of other organisms lacking obvious adaptations for dispersal can also be spread by endozoochory, with reports including midge larvae, beetle eggs, leech egg cocoons, live plantlets, and land snails (Green and Sanchez 2006, Laux and Kolsch 2014, Simonová et al. 2016, Silva et al. 2018). The low proportion of viable fish eggs we detected in experimental droppings is similar to survival rates described for other aquatic propagules ingested by waterfowl (Laux and Kolsch 2014). The annual killifish cycle coincides with peak waterfowl presence in the study region between July and October. Like other waterfowl, coscoroba swans move daily between different waterbodies separated by several kilometers to feed and roost, as well as making much longer movements during seasonal migrations (Silva et al. 2018). The retention times recorded suggest killifish eggs could be dispersed over 2,000 km during a nonstop flight (van Leeuwen et al. 2012).

The eggs of annual killifish, including our study species, are well adapted to harsh conditions (Reichard and Polačik 2019). The life cycle of annual killifish includes a period when the entire population resides in desiccated sediment of their natal pools as embryos protected in the eggs with a thick chorion (Podrabsky et al. 2017). At this stage, the embryos are protected in diapause II (Reichard and Polačik 2019) with their metabolic rates reduced by $90 \%$, and are insensitive to a harsh external environment (Podrabsky and Hand 1999). In addition, the outer layer of the chorion has complex filaments (Podrabsky and Hand 1999) that may further increase egg protection. Further research should identify how the chorion protects killifish embryos from protease digestion and the acidic environment in the waterfowl stomach.

Dispersal of killifish eggs through gut passage can explain the presence of annual killifish in isolated ephemeral pools that are not subject to flooding, and reestablishment of killifish populations after pools remain dry over multiple years (Reichard 2015). We provide evidence of endozoochory in two annual killifish species, raising the possibility that other fish species might also disperse this way. The extent to which fish species disperse by extraordinary modes such as avian gut passage is expected to be low, but our work suggests it has been overlooked and requires focused research. Current evidence of ectozoochory is based on a single anecdotal report, where viable eggs of pike (Esox lucius) were found attached to the feet of mallards (Anas platyrhynchos; Riehl 1991). Hence, as well as being important over evolutionary time scales, endozoochory may also be significant over ecological timescales, and explain surprising records of fish in isolated lakes and pools.

\section{ACKNOWLEDGMENTS}

GGS thanks CAPES for a doctoral scholarship. LM and CS hold Research Productivity Grants from CNPq. AJG was supported by Spanish National Plan project CGL2016-76067-P (AEI/FEDER, EU). MR was funded by CSF 18-26284S. Author contributions: GGS collected field samples. GGS, VW, and $\mathrm{PH}$ designed and conducted the experiment. GGS, VW, $\mathrm{PH}$, and LL collected fish. VW took care of eggs and fish in the laboratory, with contributions by PH, LL, and MV. GGS, VS, and VW conducted the experiment with birds. VW and PH prepared images. MR, AJG, GGS, and LM drafted the manuscript. All authors interpreted results and contributed to the text. LM and CS coordinated research.

\section{Literature Cited}

Bartáková, V., M. Reichard, K. Janko, M. Polačik, R. Blažek, K. Reichwald, A. Cellerino, and J. Bryja. 2013. Strong population genetic structuring in an annual fish, Nothobranchius furzeri, suggests multiple savannah refugia in southern Mozambique. BMC Evolutionary Biology 13:196.

Furness, A. I., D. N. Reznick, M. S. Springer, and R. W. Meredith. 2015. Convergent evolution of alternative developmental trajectories associated with diapause in African and South American killifish. Proceedings of the Royal Society B 282:20142189.

Green, A. J., and M. I. Sanchez. 2006. Passive internal dispersal of insect larvae by migratory birds. Biology Letters 2:55-57. 
Green, A. J., M. Soons, A. Brochet, and E. Kleyheeg. 2016. Dispersal of plants by waterbirds. Pages 147-195 in C. H. Şekercioğlu, D. G. Wenny, and C. J. Whelan, editors. Why birds matter: avian ecological function and ecosystem services. University of Chicago Press, Chicago, Illinois, USA.

Hirsch, P. E., A. N'Guyen, R. Muller, L. Adrian-Kalchhauser, and P. Burkhardt-Holm. 2018. Colonizing islands of water on dry land on the passive dispersal of fish eggs by birds. Fish and Fisheries 19:502-510.

Laux, J. J., and G. Kolsch. 2014. Potential for passive internal dispersal: eggs of an aquatic leaf beetle survive passage through the digestive system of mallards. Ecological Entomology 39:391-394.

Lovas-Kiss, Á., M. I. Sanchéz, V. A. Molnar, L. Valls, X. Armengol, F. Mesquita-Joanes, and A. J. Green. 2018. Crayfish invasion facilitates dispersal of plants and invertebrates by gulls. Freshwater Biology 63:392-404.

Podrabsky, J. E., and S. C. Hand. 1999. The bioenergetics of embryonic diapause in an annual killifish, Austrofundulus limnaeus. Journal of Experimental Biology 202:2567-2580.

Podrabsky, J. E., C. L. Riggs, A. L. Romney, S. C. Woll, J. T. Wagner, K. M. Culpepper, and T. G. Cleaver. 2017. Embryonic development of the annual killifish Austrofundulus limnaeus: an emerging model for ecological and evolutionary developmental biology research and instruction. Developmental Dynamics 246:779-801.

Reichard, M. 2015. The evolutionary ecology of African annual fishes. Pages 133-158 in N. Berois, N. G. García, and R. de
Sá, editors. Annual fishes: life history strategy, diversity, and evolution. CRC Press, Boca Raton, Florida, USA.

Reichard, M., and M. Polačik. 2019. The natural history of model organisms: Nothobranchius furzeri, an "instant" fish from an ephemeral habitat. eLife 8:e41548.

Riehl, R. 1991. Können einheimische Fische anhand ihrer Eier durch Wasservögel verbreitet werden? Zeitschrift für Fischkunde 1:79-83 [In German].

Silva, G. G., A. J. Green, V. Weber, P. Hoffmann, Á. Lovas-Kiss, C. Stenert, and L. Maltchik. 2018. Whole angiosperms Wolffia columbiana disperse by gut passage through wildfowl in South America. Biology Letters 14:20180703.

Simonová, J., O. P. Simon, Š. Kapic, L. Nehasil, and M. Horsák. 2016. Medium-sized forest snails survive passage through birds' digestive tract and adhere strongly to birds' legs: more evidence for passive dispersal mechanisms. Journal of Molluscan Studies 82:422-426.

van Leeuwen, C. H., G. van der Velde, J. M. van Groenendael, and M. Klaassen. 2012. Gut travellers: internal dispersal of aquatic organisms by waterfowl. Journal of Biogeography 39:2031-2040.

Additional supporting information may be found in the online version of this article at http://onlinelibrary.wiley.com/doi/10. 1002/ecy.2774/suppinfo 